\title{
Why we need to account for human behavior and decision-making to effectively model the non-linear dynamics of livestock disease
}

\author{
Scott C. Merrill \\ Department of Plant and Soil Science \\ Gund Institute for Environment \\ University of Vermont, Burlington, VT 05405-0082, USA \\ Gabriela Bucini \\ Department of Plant and Soil Science \\ University of Vermont, Burlington, VT 05405-0082, USA \\ Eric M. Clark \\ Department of Plant and Soil Science \\ University of Vermont, Burlington, VT 05405-0082, USA \\ Christopher J. Koliba \\ Department of Community Development and Applied Economics \\ Gund Institute for Environment \\ University of Vermont, Burlington, VT 05405-0082, USA \\ Luke Trinity \\ Department of Computer Science \\ University of Victoria, British Columbia, Canada \\ Asim Zia \\ Department of Community Development and Applied Economics \\ Department of Computer Science \\ Gund Institute for Environment \\ University of Vermont, Burlington, VT 05405-0082, USA \\ Ollin Langle-Chimal \\ Department of Computer Science \\ Complex Systems Center \\ University of Vermont, Burlington, VT 05405-0082, USA \\ Nicholas Cheney \\ Department of Computer Science \\ Gund Institute for Environment
}




\title{
Complex Systems Center \\ University of Vermont, Burlington, VT 05405-0082, USA
}

\author{
Trisha R. Shrum \\ Department of Community Development and Applied Economics \\ University of Vermont, Burlington, VT 05405-0082, USA
}

\author{
Timothy Sellnow \\ Nicholson School of Communication \\ University of Central Florida, Orlando, FL 32816-1344, USA. \\ Deanna Sellnow \\ Nicholson School of Communication \\ University of Central Florida, Orlando, FL 32816-1344, USA. \\ Julia M. Smith \\ Department of Animal and Veterinary Sciences \\ University of Vermont, Burlington, VT 05405-0082, USA
}

\begin{abstract}
Animal disease costs the livestock industries billions of dollars annually. These costs can be reduced using effective biosecurity. However, costs of biosecurity are steep and benefits must be weighed against the uncertain infection risks. Much effort has gone into determining efficacy of different biosecurity tactics and strategies. Unfortunately, the variability in human behavior and decision-making when confronted with risk information has largely been overlooked. Here we show that use of the human behavioral component is necessary to understand the patterns of infection incidence in livestock industries. Using an agent-based model developed with a foundation of supply chain and industry structural data, we integrate human behavioral data generated using experimental games that parameterizes communication strategies, learning, psychological discounting and categorization of human behavior along a risk aversion spectrum. The influence of risk communication strategies on human behavior can be tested with experimental gaming simulations and their impact on the system can be projected using agent-based models, delivering feedback to increase disease resiliency of production systems.
\end{abstract}

Keywords - Agent-Based Model, Biosecurity, Experimental Game, Risk Aversion, Risk Communication

SUGGESTED CITATION: Merrill, S. C., Bucini, G., Clark, E. M., Koliba, C.J., Trinity, L., Zia, A., Langle-Chimal, O., Cheney, N., Shrum, T. R., Sellnow, T., Sellnow, D. D., \& Smith, J.M. (2021). Why we need to account for human behavior and decision-making to effectively model the non-linear dynamics of livestock disease. Proceedings of the International Crisis and Risk Communication Conference, Volume 4 (pp. 23-28). Orlando Fl: Nicholson School of Communication and Media. https://doi.org/10.30658/icrcc.2021.06

\section{INTRODUCTION}

Livestock disease in the U.S. results in significant animal welfare issues and economic loses in the billions every year. In swine production, endemic diseases such as Porcine Reproductive and Respiratory Syndrome and Porcine Epidemic Diarrhea virus (PEDv) cost well over a billion dollars annually [1]. Epidemiological research highlights vectors of spread and efficacy of biosecurity practices (i.e., practices designed to reduce the spread of disease). Yet, epidemiological and disease models used to understand the spread and impact of disease rarely incorporate prevention-related human behavior or decision-making. Here, we acknowledge that biosecurity is enacted by people, animals are transported through human networks, and disease is frequently vectored through mechanisms controlled by people. As such, the decisions and behavior of individuals that interact with the animals, support the industry, and work in the supply chain may be key drivers of disease spread and impact. 
PEDv, which serves as our primary disease example, was first detected in the U.S. in May 2013 and spread rapidly across the swine industry (Fig. 1). After an initial surge in the winter of 2013-2014, virus incidence subsided during the summer and fall before resurging again in the winter of 20142015. This cycle repeated with peak annual incidence diminishing over time (green line in Fig. 1) [2]. During this time period, PEDv did not notably evolve, rather the decrease in incidence was associated with a behavioral response, and the resultant improved biosecurity.

Gathering data on human behavior and biosecurity decisions is challenging because it is difficult to determine, solely through traditional methods like surveys, how individuals might respond to different outbreak scenarios [3]. Generating realistic representations of behavior may be enhanced by buttressing traditionally acquired data with experimental game data. Experimental games can be used to simulate environments, thus allowing for collecting behavioral response data that would otherwise be impossible to obtain. Data gathered using experimental games can be used either directly to help understand the variability and trends in behavior, or they may be used as inputs into complex models, for example, to help predict aspects of social-ecological systems.

One approach to modeling complex systems is through the use of agent-based modeling. Real world systems can be emulated with agent-based models (ABMs) using a bottom-up approach where a collection of autonomous decisionmaking entities called agents are involved in spatiotemporal processes. The dynamic patterns emerging at the system level from the agents' behavior and interactions can be captured in output variables and analyzed statistically. ABM inputs include fixed parameters, as well as stochastic variables, in this case probabilistic disease transmission, and location of different types of facilities. Network arrangements were randomized allowing for variation in feed delivery, and moving animals for processing, with ramifications for disease transmission.

Our research sought to parameterize the effects of human behavior on disease transmission in the swine industry, including examining potential ramifications of altering communication strategy and to test the overarching hypothesis: Human behavior and decision-making can alter the trajectory of a disease incursion, significantly influencing livestock health and economic consequences. Data collection was completed in multiple phases and integrated into an animal disease biosecurity agent-based model.

\section{METHODS}

We used experimental games, surveys and workshops to gather data on human decision-making behavior in response to simulated disease outbreak scenarios. To gather experimental game data, we recruited thousands of participants from a variety of communities ranging from on-campus university communities, to swine industry specialists and veterinarians, and participants from an online workforce (Fig. 2).

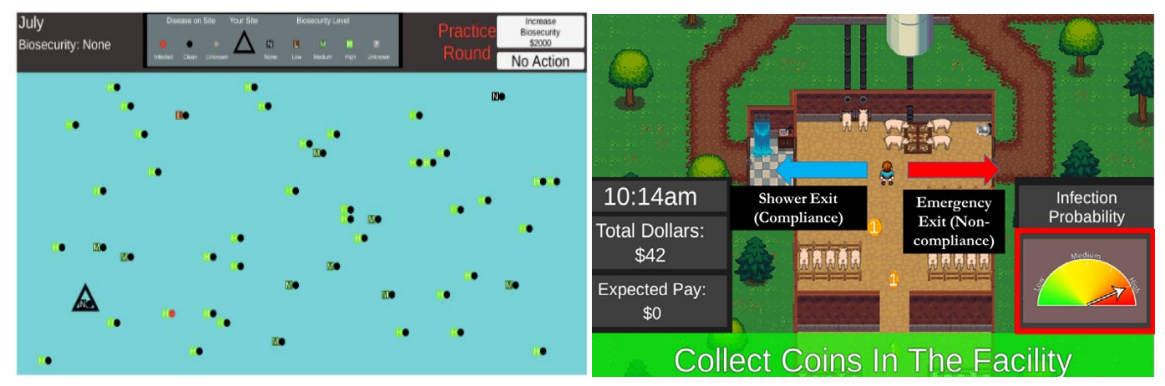

Figure 2. Screenshots of our experimental games. Left: An experimental game examining the willingness to invest in preventative health tactics $[4,5]$. Right: an experimental game examining willingness to follow animal health protection rules to prevent disease incursions $[6,7]$.

The agent-based model was developed using USDA industry specific data, industry-provided supply chain data as well as data derived from experimental game results. To model the spread of disease among facilities, the ABM used a susceptible-infected-susceptible epidemiological model (Fig. 3). 


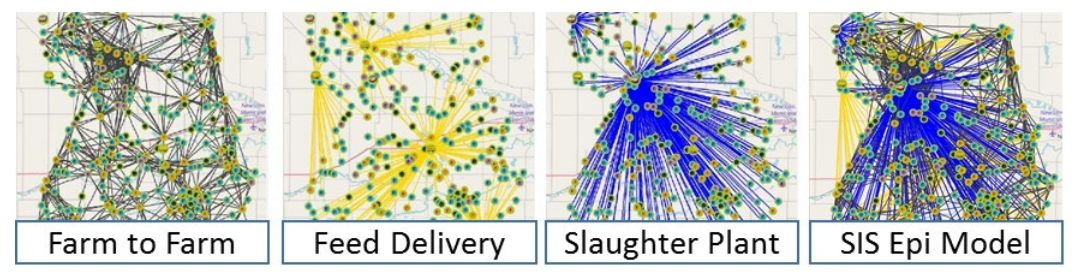

Figure 3. The calibrated ABM used autonomous agents acting in a networked supply chain and was parameterized with USDA, industry supply chain, expert opinion and experimental game data. Disease spread was simulated using a Susceptible-Infected-Susceptible epidemiological (SIS Epi) model. Human behavior characteristics were added into the model structure and influenced the probability of disease transfer via biosecurity decision-making $[2,8]$.

\section{RESULTS}

Data from the experimental games provided both insight about human behavior in situations of disease risk as well as input data for the ABM. Risk messages increase in efficacy from poor efficacy using numbers to good efficacy using graphical displays or infographics (Figure 5) [6]. When considering disease risk communication strategy, reporting information about incidence to the game participants instead of withholding it prompted increased willingness in participants to invest in biosecurity. Conversely, withholding information about biosecurity tactics adopted by others within the industry increased willingness to invest in biosecurity. These results suggest that information sharing will promote different responses depending upon the information type [5]. Responses to risk situations varied along a spectrum of risk aversion. This allowed for individuals to be categorized as largely attempting to avoid risk, tolerant of risk or those that moved along the risk aversion spectrum depending upon the situation [4]. Moreover, many participants learned, and adjusted their strategy over the course of the experiment, with some becoming more risk averse and others becoming more risk tolerant. Interestingly, behavioral responses did not vary substantially between those in the industry and a broad audience recruited using the online workforce Amazon Mechanical Turks [3]. We found that we were able to shift or nudge participants along the risk spectrum by altering how disease risk messages were delivered, including in some situations doubling or tripling the willingness to practice biosecurity, thus dramatically reducing risky behavior.

Behavioral data and modeled trends were incorporated into the ABM in multiple ways, which allowed for simulation of complexity in human response to disease outbreak scenarios. Psychological discounting, which was observed during the experiments and noted in focus groups with industry specialists, was parameterized and included in the ABM. Inclusion of psychological discounting behavior allows for simulated workers on the ground to become increasingly lax over time if they have not observed a disease incursion. Variability in willingness to invest in biosecurity in response to disease risk was parameterized using behavioral clusters with simulated behavior of each cluster dependent upon risk situation and estimated risk aversion. Specifically, mirroring the experimental-game finding, awareness of increased disease incidence in the system led agents to increase their biosecurity investments. Finally, learning behaviour was incorporated in the ABM, which allowed for subtle behavioral shifts in risk aversion by agents in response to disease outbreak situations.

\section{DISCUSSION}

This work builds on research suggesting that human behavior is highly variable, and decidedly irrational. Motivational factors are complex and difficult to determine. While predictions of individual behavior are fraught with error, we can with some consistency, determine the distribution of individuals along a risk aversion spectrum. In general, messages for conveying disease risk information that include numbers (e.g., infection risk as a percentage) resulted in reduced willingness to follow biosecurity rules compared to using simple word phrases. Higher than numbers or word phrases was willingness to follow rules when information was provided using graphical threat gauges. Tendencies towards increased investment were observed when we used communication strategies that provided complete information about disease incidence. Conversely, we observed increased investment when we withheld information about what other people and facilities were doing to prevent disease outbreaks (i.e., biosecurity practices used at facilities). Thus, sometimes providing information was beneficial while sometimes withholding information led to increased investment. One distinction between these two types of information is that knowing what others are doing to prevent disease allows for free-rider behavior, whereas knowing where disease risk is high does not. These findings have interesting implications for vaccine hesitancy and should be further examined to determine if open information flow may lead to the perverse reduction in vaccine use.

We were able to cluster behaviors along a risk spectrum and recognized three main responses to disease risk namely aversion, tolerance, and a group that displayed high situational awareness and varied their behavior based on the risk environment. In our modeling work, we parameterized the behavioral rules of the ABM agents using three types of responses: 1) Willingness to invest in biosecurity when confronted by risk. 2) Potential shifts in behavioral tendencies 
over time (learning) and 3) psychological discounting. Thus, rational and irrational human behaviour and variability was coded into modeled agents in the swine industry supply chain network. We calibrated the ABM using observed PEDv incidence and then used the calibrated ABM to simulate disease spread. Calibrated ABM model runs allowed us to create envelopes of possible disease outbreak scenarios, and thus, observe emergent disease impact patterns stemming from the coupled effects of epidemiological mechanisms and human behavior. Moreover, we could simulate alternative communication strategies, and observe resultant changes to the system.

Thousands of experiments were run using the calibrated ABM and varying human behavioral components. Critically, we were unable to match the observed decreasing trend in PEDv disease incidence (Fig. 1) without including human behavior and decision-making. The best fitting ABM was generated using a starting population that was largely risk tolerant. If the ABM was parameterized using a dominantly risk averse population, we observed rapid suppression of the disease, whereas with $100 \%$ risk tolerant populations (or populations that were naïve to effective biosecurity tactics for the suppression of the disease) substantial outbreaks were regularly observed, but with high variability in duration and impact, ranging from quick suppression to epidemics (Fig. 4).

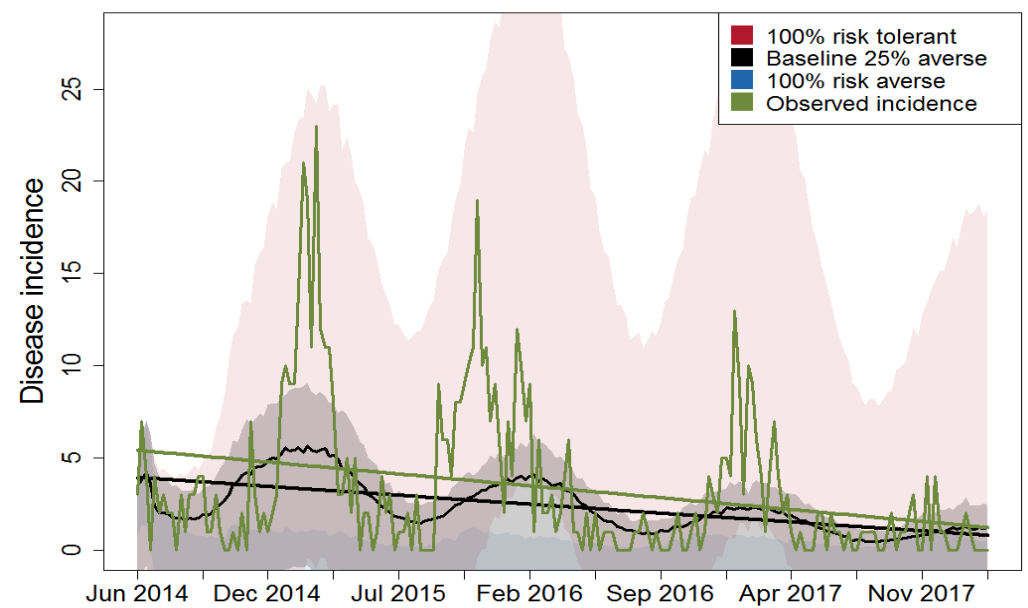

Figure 4. PEDv observed incidence (green) decreases over time (green trend line) which can only be matched by a model that incorporates a human response (Black trend line and shading of well-fit model predictions) (Bucini et al. Forthcoming). Switching communication strategy can shift outbreaks from epidemic level (pink shading depicts model runs with risk tolerant populations) to quickly suppressed (Risk averse populations quickly shut down disease outbreaks as depicted by blue shading).

\section{CONCLUSION}

Epidemiological models are unable to capture the reduction in PEDv incidence without incorporating human behavioral responses to the outbreak. Risk communication strategy can dramatically influence willingness to practice and invest in disease prevention tactics, with effects shaping the impact of the disease outbreak severity. Effective disease messaging and communication strategies have the potential to positively influence risk reduction behavior, resulting in quick disease suppression. Conversely, ineffective messaging leads to high average disease impacts but with a wide confidence interval because of the exceptionally high variability in disease outbreak severity, meaning that the system was left vulnerable to high unpredictability in the face of disease outbreaks - sometimes the disease was quickly suppressed and other times reached epidemic levels. Overall, we show that behavior and decision-making is a critical component for disease modeling, without which models cannot capture observed disease trends.

\section{Author Biography}

Scott C. Merrill, (Ph.D. Colorado State University, Fort Collins, Colorado) Department of Plant and Soil Science, University of Vermont, Burlington, VT 05405, U.S.A. Email: Scott.C.Merrill@uvm.edu

Gabriela Bucini, (Ph.D. Colorado State University, Fort Collins, Colorado) Department of Plant and Soil Science, University of Vermont, Burlington, VT 05405, U.S.A.

Eric M. Clark, (Ph.D. University of Vermont, Burlington, Vermont) Department of Plant and Soil Science, University of Vermont, Burlington, VT 05405, U.S.A.

Christopher J. Koliba (Ph.D. Syracuse University, Syracuse, New York) Department of Community Development and Applied Economics, University of Vermont, Burlington, VT 05405, U.S.A.

Luke Trinity (M.S. University of Vermont, Burlington, Vermont) Department of Computer Science, University of Victoria, British Columbia, Canada

Asim Zia (Ph.D. Georgia Institute of Technology, Atlanta, Georgia) Department of Community Development and Applied Economics and Department of Computer Science, University of Vermont, Burlington, VT 05405, U.S.A 
Ollin Langle-Chimal (M.S. Instituto Tecnológico Autónomo de México, Mexico City, Mexico) Department of Computer Science, University of Vermont, Burlington, VT 05405, U.S.A.

Nicholas Cheney (Ph.D., University of Vermont, Burlington, Vermont) Department of Computer Science, University of Vermont, Burlington, VT 05405, U.S.A.

Trisha R. Shrum (Ph.D., Harvard University, Cambridge, Massachusetts) Department of Community Development and Applied Economics and Department of Computer Science, University of Vermont, Burlington, VT 05405, U.S.A

Timothy Sellnow (Ph.D. Wayne State University, Detroit, Michigan), Nicholson School of Communication, University of Central Florida, Orlando, FL 32816-1344, U.S.A.

Deanna D. Sellnow (Ph.D., The University of North Dakota, Grand Forks, North Dakota), Nicholson School of Communication, University of Central Florida, Orlando, FL 32816-1344, U.S.A.

Julia M. Smith (Ph.D., Cornell University), Department of Animal and Veterinary Sciences, University of Vermont, Burlington, VT 05405, USA.

Acknowledgement: This work is supported by the USDA National Institute of Food and Agriculture, under award number 2015-69004-23273. The contents are solely the responsibility of the authors and do not necessarily represent the official views of the USDA or NIFA. (Wiltshire et al., 2019)

\section{REFERENCES}

[1] Holtkamp, D. J., Kliebenstein, J. B., Neumann, E. J., Zimmerman, J. J., Rotto, H. F., Yoder, T. K., Wang, C., Yeske, P. E., Mowrer, C. L., \& Haley, C. A. (2013). Assessment of the economic impact of porcine reproductive and respiratory syndrome virus on United States pork producers. Journal of Swine Health and Production, 21(2), 72-84.

[2] Bucini, G., Merrill, S. C., Clark, E., Moegenburg, S. M., Zia, A., Koliba, C. J., Wiltshire, S., Trinity, L., \& Smith, J. M. (2019). Risk attitudes affect livestock biosecurity decisions with ramifications for disease control in a simulated production system. Frontiers in Veterinary Science, 6(196). https://doi.org/10.3389/fvets.2019.00196

[3] Clark, E. M., Merrill, S. C., Trinity, L., Bucini, G., Cheney, N., Langle-Chimal, O., Shrum, T., Koliba, C., Zia, A., \& Smith, J. M. (2021). Emulating Agricultural Disease Management: Comparing Risk Preferences between Industry Professionals and Online Participants Using Experimental Gaming Simulations and Paired Lottery Choice Surveys. Frontiers in Veterinary Science, 7, 556668. https://doi.org/10.3389/fvets.2020.556668

[4] Clark, E. M., Merrill, S. C., Trinity, L., Bucini, G., Cheney, N., Langle-Chimal, O., Shrum, T., Koliba, C. J., Zia, A., \& Smith, J. M. (2020). Using experimental gaming simulations to elicit risk mitigation behavioral strategies for agricultural disease management. Plos One, 15(3) e022898. https://doi.org/10.1371/journal.pone.0228983

[5] Merrill, S. C., Koliba, C. J., Moegenburg, S., Zia, A., Parker, J., Sellnow, T., Wiltshire, S., Bucini, G., Danehy, C., \& Smith, J. M. (2019). Decision-making in livestock biosecurity practices amidst environmental and social uncertainty: Evidence from an experimental game. Plos One, 14(4) e214500. https://doi.org/10.1371/journal.pone.0214500

[6] Merrill, S. C., Moegenburg, S., Koliba, C. J., Zia, A., Trinity, L., Clark, E. M., Bucini, G., Wiltshire, S., Sellnow, T., Sellnow, D. D., \& Smith, J. M. (2019). Willingness to comply with biosecurity in livestock facilities: Evidence from experimental simulations. Frontiers in Veterinary Science, 6(156). https://doi.org/10.3389/fvets.2019.00156

[7] Trinity, L., Merrill, S. C., Clark, E. M., Koliba, C. J., Zia, A., Bucini, G., \& Smith, J. M. (2020). Effects of Social cues on biosecurity compliance in livestock facilities: Evidence from experimental simulations. Frontiers in Veterinary Science, 7(130). https://doi.org/10.3389/fvets.2020.00130

[8] Wiltshire, S., Zia, A., Koliba, C., Bucini, G., Clark, E., Merrill, S. C., Smith, J. M., \& Moegenburg, S. (2019). Network meta-metrics: Using evolutionary computation to identify effective indicators of epidemiological vulnerability in a livestock production system model. Jasss-the Journal of Artificial Societies and Social Simulation, 22(2), 27. https://doi.org/10.18564/jasss.3991 